\title{
GEORREFERENCIAMENTO DE IMÓVEIS RURAIS: ANÁLISE DO CÁLCULO DE ÁREA
}

\author{
Bruno Zucuni Prina ${ }^{1}$ \\ Romario Trentin ${ }^{2}$ \\ Renato Giovani Chaves de Sá ${ }^{3}$ \\ Luiz Felipe Díaz de Carvalho ${ }^{4}$
}

Resumo: Neste trabalho foi desenvolvida uma metodologia para análise do cálculo de área, com coordenadas localizadas em três regiões do fuso UTM ( $k=1, k>1, k<1)$. Dessa forma, objetivou-se em realizar a conversão de coordenadas geográficas para locais e, ainda, comparar as áreas obtidas em coordenadas UTM e locais. Para confecção da metodologia, criaram-se dois aplicativos, por meio da linguagem de programação Visual Basic, a fim de formatar as coordenadas originais e salvá-las em um banco de dados para que assim, posteriormente, as mesmas fossem convertidas (de geográficas para locais) e, posteriormente, realizar o cálculo da área da poligonal (em local). Notou-se que o cálculo de área a partir das coordenadas UTM mostra-se incoerente para com a realidade topográfica local, pois, dependendo de sua localização espacial no fuso UTM, as variações tendem a serem maiores ou menores. Ainda, constatou-se que, apesar da pequena variação de área constatada, é importante salientar que as precisões posicionais (para um vértice) estabelecidas pelo INCRA são mínimas, logo, é importante analisar e discutir as pequenas variações superficiais, as quais discriminam diferenças na área da poligonal.

Palavras-chave: Coordenadas Locais; INCRA; SIGEF; UTM; Algoritmos; Visual Basic.

\section{GEOREFERENCING OF RURAL PROPERTY: ANALYSIS OF CALCULATION OF AREA}

Abstract: In this paper we develop a methodology for analyzing the area calculation, with coordinates located in three regions of the zone UTM $(k=1, k>1, k<1)$. Thus, the purpose of this paper is to realize the conversion of geographic coordinates to local coordinates, and also comparing the areas obtained in UTM coordinates and local coordinates. For the methodology of this paper, we build two apps with the Visual Basic programming language, in order to format the original coordinates and save them on a database so that, later they be converted (geographic for local) and, subsequently, to perform the calculation of the area of the polygon (local coordinates). We realized that the area calculation from UTM coordinates is shown to inconsistent with local topographic reality, on this account, depending on your spatial location the UTM zone, the variations tend to be larger or smaller.

1. Mestrando em Geografia, Tecnólogo em Geoprocessamento, Técnico em Geomática; Universidade Federal de Santa Maria (UFSM) (brunozprina@gmail.com).

2. Doutor de Geografia, Professor da UFSM; Universidade Federal de Santa Maria (UFSM) (romario.trentin@gmail.com).

3. Técnico em Geoprocessamento; Universidade Federal de Santa Maria (UFSM) (renas.leny@hotmail.com).

4. Agrônomo, Professor da UFSM; Universidade Federal de Santa Maria (UFSM) (Ifelipe.dc@gmail.com). 
Also, it was found that, despite the small area variation, is important to contrast which positional precisions (for a vertex) established by INCRA are minimal, ergo, is important to analyze and discuss small surface variations, which discriminate differences in the polygonal area.

Key words: Coordinates Local, INCRA, SIGEF, UTM, Algorithms, Visual Basic.

\section{INTRODUÇÃO}

Desde a primeira edição da Norma Técnica do Georreferenciamento de Imóveis Rurais (NTGIR) (INCRA, 2003), até a 2ª edição revisada (INCRA, 2010b), que estava em vigor até o final de 2013, o cálculo da área (da poligonal) de uma propriedade rural, com o intuito de certificação junto ao Instituto Nacional de Colonização e Reforma Agrária (INCRA), era gerado sobre o plano da projeção Universal Transversa de Mercator (UTM). Tal método altera o valor da área da poligonal, principalmente nas zonas de redução e ampliação de um fuso UTM, fato interligado às deformações do plano UTM. Hoje, com a 3a NTGIR (INCRA, 2013a) o cálculo de área é realizado por intermédio das coordenadas locais, localizado sobre um Sistema Geodésico Local (SGL), as quais são obtidas pelas coordenadas geográficas (latitude, longitude e altitude elipsoidal) ou cartesianas $(X, Y, Z)$, recorrendo a uma matriz de rotação ortogonal para realização da conversão.

Por meio da portaria no 486 de 2 de setembro de 2013 (BRASIL, 2013), houve a homologação da 3ạ Edição da NTGIR (INCRA, 2013a), do Manual Técnico de Posicionamento (INCRA, 2013b) e do Manual Técnico de Limite e Confrontações (INCRA, 2013c). Com a referida norma técnica e seus anexos, houve uma acentuada mudança no âmbito do georreferenciamento no Brasil, pois o processo de certificação dos imóveis rurais passou a ser totalmente informatizado, utilizando, como meio de certificação o Sistema de Gestão Fundiária (SIGEF, 2014).

Assim, a 3a edição da NTGIR (INCRA, 2013a), é composta por alguns documentos auxiliares, que são destacados a seguir. O "Manual para Gestão da Certificação de Imóveis Rurais", o qual contém todos os procedimentos metodológicos que subsidiam a norma técnica, destacando as formas de encaminhamentos de todas as ações de certificação dos imóveis, bem como da conduta correta para prováveis desmembramentos e remembramentos, cancelamentos e retificações, entre outros procedimentos (INCRA, 2013d). A estruturação 
do processo de certificação ainda passa pelo "Manual Técnico de Limites e Confrontações" (INCRA, 2013c) que destaca, principalmente, os tipos de vértices que a poligonal pode possuir e os tipos de limites. O "Manual Técnico de Posicionamento" (INCRA, 2013b), por sua vez, tem uma importância significativa para esse trabalho, pelo fato de que o mesmo procede da explicação de como realizar o cálculo de área das poligonais, diferenciando dos demais procedimentos (de cálculo de área) das outras edições da NTGIR (INCRA, 2003, 2010a, 2010b). No entanto, atualmente, no referido manual, a área é referenciada no SGL, e os resultados obtidos, por meio desse processo, explicitam melhor a realidade física. Assim, eliminam-se as distorções, as quais se tornam maiores na medida em que as parcelas aumentam sua superfície (INCRA, 2013b). Para um melhor entendimento do referido processo, tem-se, no referido manual, a explanação de como realizar a conversão para coordenadas locais, na qual é utilizada uma matriz de rotação ortogonal. Ainda, na Figura 1, há a ilustração de como é sistematizado o SGL, com a demonstração dos eixos cartesianos.

Conforme informações de Dal'forno et al. (2011), no sistema de coordenadas terrestres local, é permitido realizar implantações de obras, com alta precisão, sendo uma grande solução técnica para a os projetos que envolvam engenharia e cadastro. Ainda, Dal'forno et al. (2011) complementam que a conversão das coordenadas geodésicas para locais "é de suma importância para facilitar o seu emprego e ao aperfeiçoamento da normalização dessas transformações, de modo que, a exatidão seja compatível com as especificações estabelecidas".

Sequencialmente são contextualizadas algumas definições que são os pilares, para que sejam constatados, alguns procedimentos metodológicos do presente trabalho. Assim, destacam-se os conceitos de algoritmos, coordenadas geográficas, UTM, cartesianas e locais. Um algoritmo é expresso por meio da aplicação de uma lógica matemática e é expressa por meio de uma determinada linguagem de programação (BATALHA, 2008), e é expressa por meio de uma determinada linguagem de programação. Ximenes (1993 apud BATALHA, 2008, p. 14) define o conceito de algoritmo, sendo necessárias duas visões. A primeira referente a um conceito mais simples, estabelecendo relações do cotidiano das pessoas, sugerindo a ideia de um "conjunto finito de instruções que possa ser seguido para a realização de uma tarefa específica ou resolução de determinado problema". A segunda visão exposta por Ximenes (1993 apud BATALHA, 2008) é expresso o sentido de algoritmo dentro da 
programação, entendendo que o mesmo é caracterizado por ser uma estrutura ordenada e finita de procedimentos a fim de atingir a solução de um determinado problema.

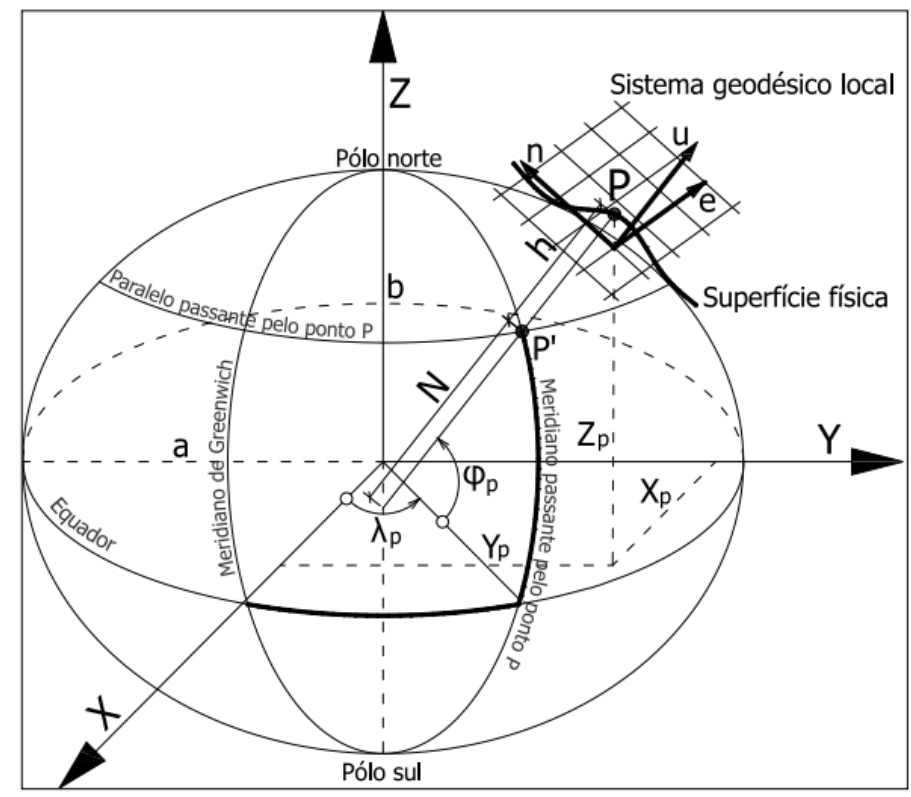

Figura 1: Sistema Geodésico Local e Sistema Geocêntrico. Fonte: INCRA (2013b).

Referente aos tipos de coordenadas destaca-se que, segundo Sampaio (2005) a utilização das coordenadas geográficas se faz extremamente importante visto a grande vantagem em efetivar a localização de qualquer ponto sobre a superfície terrestre. A mesma autora ainda salienta que tais coordenadas são medidas angulares, podendo, assim, ser expressas em graus decimais ou de forma sexagesimal (grau, minuto e segundo), representadas em latitude (norte e sul) e longitude (leste e oeste), variantes, por meio dos meridianos e paralelos.

Para entendimento das coordenadas UTM vale destacar que a mesma está presente numa distribuição de fusos (no total de 60) espaçados em $6^{\circ}$ de longitude, totalizando os $360^{\circ}$ da superfície terrestre. Ainda, no sentido da latitude, os mesmos estão divididos em zonas, com espaçamento de $8^{\circ}$.

As coordenadas cartesianas tridimensionais são caracterizadas por serem constituídas por três eixos cartesianos, ortogonais (ângulo de $90^{\circ}$ ) entre si, sendo representado pelas letras $\mathrm{X}$, Y e Z. Ainda, infere-se que a origem desse sistema coincide com o centro de massa da Terra; assim sendo, caracteriza-se por ser um sistema geocêntrico (MONICO, 2007). 
Por meio da metodologia de Dal'Forno et al. (2010, p. 1) destaca-se que o plano local “indubitavelmente é o que permite operacionalizar com maior exatidão a implantação de obras e de plantas cadastrais".

Assim, diante deste contexto, o referido trabalho suscitou a elaboração de dois aplicativos, a saber: a) um secundário, denominado "FormaCoo" (PRINA, 2014a), útil para a "formatação" adequada das coordenadas geográficas (em graus decimais), e posterior armazenamento das mesmas em um banco de dados, de forma automática; b) outro, principal ao referido trabalho, denominado de "ParaLocal" (PRINA, 2014b), o qual teve o objetivo de converter as coordenadas originais (geográficas decimais) para locais, e, ainda, realizando o cálculo de área da poligonal. A vantagem da utilização do aplicativo ParaLocal (PRINA, 2014b), reside no cálculo da área do polígono em função da conversão das coordenadas a partir de um "vértice-base" (o qual é definido pelo usuário, mediante escolha). Sobreleva-se que os referidos aplicativos foram estruturados por meio da linguagem de programação Visual Basic, desenvolvidos no software Microsoft Visual Basic 6.0. Para estruturar os algoritmos, fez-se uso da metodologia de cálculo de área pela Regra de Sarrus, a qual se utiliza do uso de determinantes para quantificar a área (GALVÃO, 2013).

Recentemente, foi desenvolvida uma pesquisa, visando estruturar uma rotina de conversão de coordenadas de UTM para topográfico local e vice-versa, considerando a proximidade do fuso UTM, conforme relatado em Silva et al. (2013). No entanto, não tratou da problemática do cálculo de área. Com isso, o problema desse trabalho está contido na resolução de duas questões:

- "Quais as diferenças de área envolvidas no uso das coordenadas locais com distintos vértices-base?"

- "Qual a variação da área, ao utilizar as coordenadas UTM, ao longo de um fuso, nas zonas de ampliação, redução e k=1, e quais as divergências comparando-as com as áreas obtidas pelas coordenadas locais?"

Justifica-se, desse modo, gerar uma discussão sobre a comparação de áreas localizadas em diferentes regiões de um fuso UTM, pois visto a incidência das distorções, das coordenadas UTM, às mesmas acabam por caracterizar uma área diferente para a que está contida, de fato, no campo. Além do mais, o presente trabalho justifica-se pela importância de realizar 
uma avaliação criteriosa quanto às diferenças geradas na área superficial, perante o cálculo com coordenadas UTM e locais.

O objetivo geral do trabalho é o de realizar a conversão de coordenadas geográficas para locais em três diferentes regiões do fuso UTM e comparar as áreas obtidas em coordenadas UTM e locais. Especificamente tem-se o objetivo de:

- Criar um aplicativo para formatação das coordenadas brutas (de coordenadas sexagesimais para graus decimais), bem como armazená-las em um banco de dados;

- Desenvolver um aplicativo que realize a transformação de coordenadas geográficas para locais e calcule a área da poligonal;

- Avaliar as diferenças de área ao calculá-la com as coordenadas locais em diferentes regiões do fuso UTM e com diferentes pontos-base (utilizando a matriz de rotação ortogonal); e,

- Analisar as diferenças de área entre o cálculo com coordenadas UTM e locais em diferentes regiões do fuso UTM.

\section{MATERAIS E MÉTODOS}

A fim de identificar os procedimentos teórico-práticos envolvidos, os mesmos são listados a seguir, junto com a caracterização dos aplicativos utilizados.

Destaca-se, que na etapa inicial do trabalho, compreende-se da seleção das situações das áreas, conforme a proposição do trabalho; a seguir reconheceram-se as áreas que estivessem localizadas próximas ao centro do fuso UTM (zonas de redução), nas bordas (zonas de ampliação) e nos locais com deformação nula (com fator de escala igual a um, k=1), como mostrado na Figura 2. 


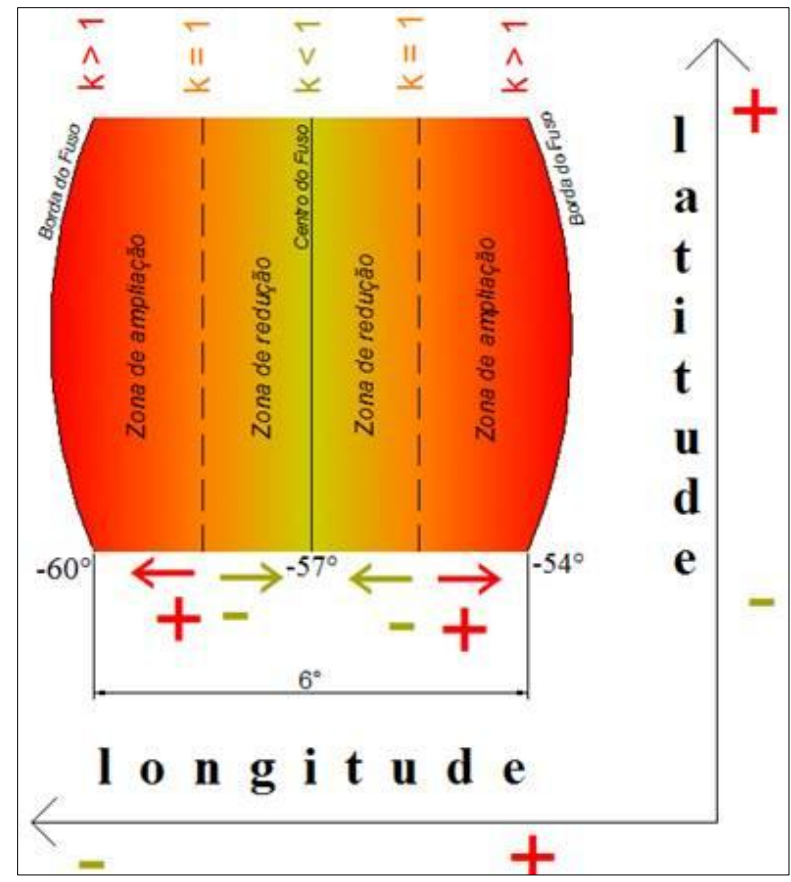

Figura 2: Análise da estrutura de um fuso UTM: Exemplificação para o fuso UTM 21 J Sul, Meridiano Central: $57^{\circ}$.

Fonte: Bruno Z. Prina (2014).

A Figura 3 sistematiza os locais onde os pontos das poligonais foram coletados, abrangendo os municípios de Uruguaiana $(k<1)$, Joia $(k>1)$ e São Francisco de Assis $(k=1)$, todos localizados no Fuso UTM 21 J Sul, no estado do Rio Grande do Sul.

Entre os aplicativos utilizados, destaca-se o FormaCoo (PRINA, 2014a), responsável pela conversão das coordenadas geográficas sexagesimais para decimais, e posterior armazenamento, de forma automática, em um banco de dados do Access ${ }^{\circledR}$ (MICROSOFT, 2007b), conforme exemplifica a Figura 4.

Dessa forma, com a utilização do aplicativo FormaCoo (PRINA, 2014a), os dados foram armazenados automaticamente e no formato correto no banco de dados do Access ${ }^{\circledR}$ (MICROSOFT, 2007b) (Figura 5). Finalmente, recorreu-se ao aplicativo ParaLocal (PRINA, 2014b), no qual foi realizado a conversão das coordenadas e o cálculo de área. 


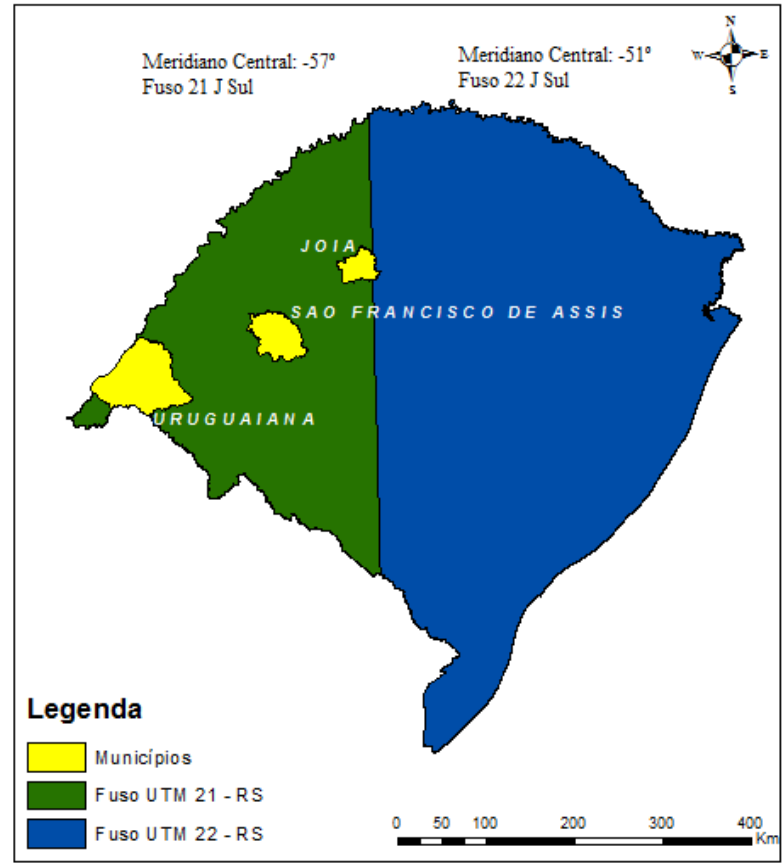

Figura 3: Localização dos dados brutos de campo. Fonte: Bruno Z. Prina (2014).

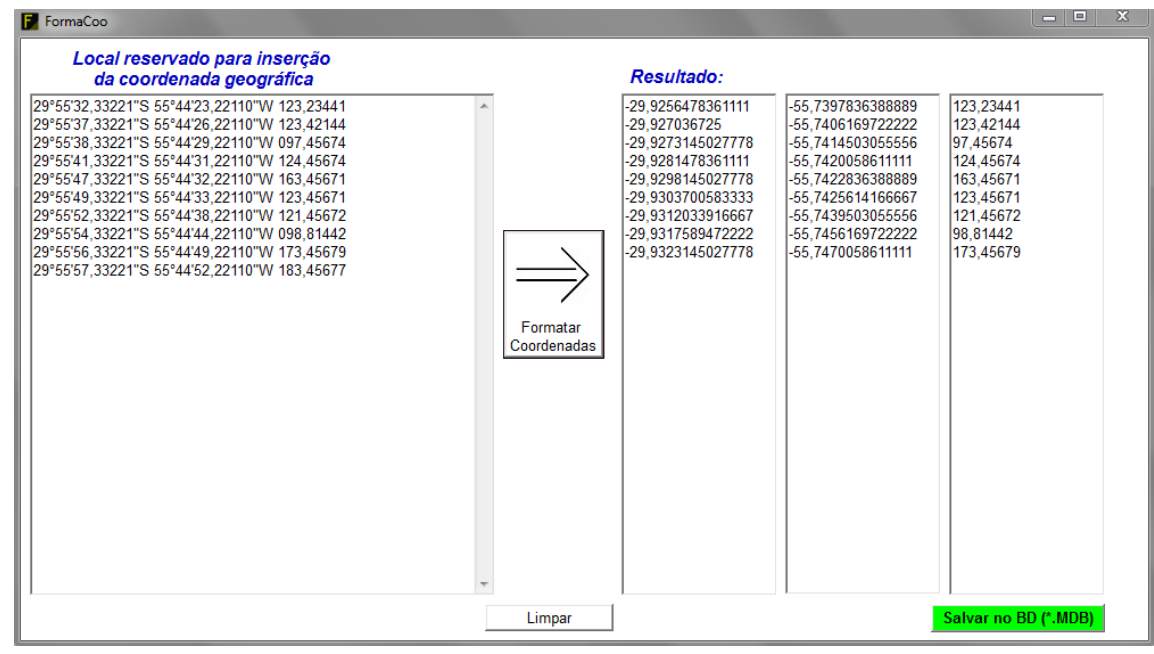

Figura 4: Visão do aplicativo FormaCoo (PRINA, 2014a). Fonte: Bruno Z. Prina (2014a).

Para manipular com excelência o aplicativo FormaCoo (PRINA, 2014a), o usuário deverá inserir as coordenadas de forma correta, com a seguinte estrutura: a latitude e a longitude deverão possuir 5 casas decimais na casa dos segundos (sendo preenchidas com zero quando não possuírem), a altitude também deverá obedecer o número de 5 casas decimais, as demais especificações quanto a formatação das coordenadas está representado na Figura 6. Outra observação, de extrema importância, é que a cada trabalho realizado o usuário deverá deixar o banco de dados vazio (sem dados), pois essa é a condição de funcionamento do FormaCoo (PRINA, 2014a). Deve-se enfatizar, ainda, que o padrão de entrada dos dados 
foi estruturado conforme o Relatório de Processamento exportado pelo Topcon Tools ${ }^{\circledR}$ (TOPCON TOOLS, 2014), aplicativo que foi utilizado no pós-processamento dos dados nativos de campo (coletados por meio da tecnologia GNSS).

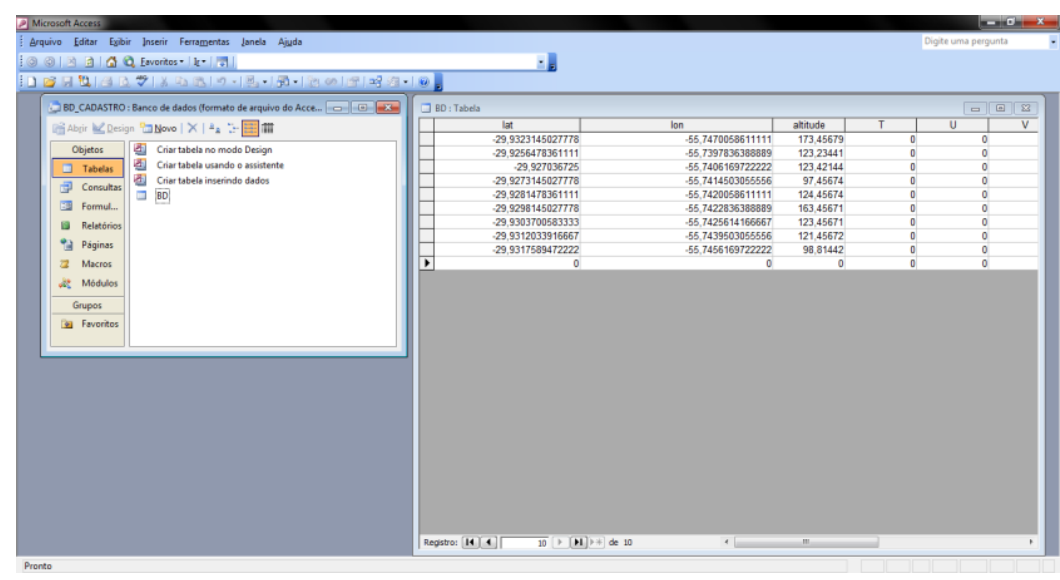

Figura 5: Organização do Banco de Dados. Fonte: Bruno Z. Prina (2014).

\section{GGंMM'SS,sssss"S GG MM'SS,sssss"W HHH,hhhhh latitude longitude altitude}

Figura 6: Formato de entrada das coordenadas ao FormaCoo (PRINA, 2014a). Fonte: Bruno Z. Prina (2014).

Para manipulação do aplicativo ParaLocal (PRINA, 2014b), primeiramente, definiu-se o datum horizontal (ou sistema de referência) dos dados nativos, conforme a Figura 7, cujo exemplo apresenta o "SIRGAS 2000". A seguir acionou-se o botão "Abrir Banco de Dados", (Figura 7) e selecionou-se o arquivo que anteriormente foi organizado em formato de tabela (*.MDB, que no exemplo da mesma figura corresponde ao campo em cinza "C: $\mid$ modelo. $m d b^{\prime}$ ") conforme o padrão evidenciado na Figura 5 (apresentada anteriormente), na qual é sistematizada automaticamente pelo FormaCoo (PRINA, 2014a).

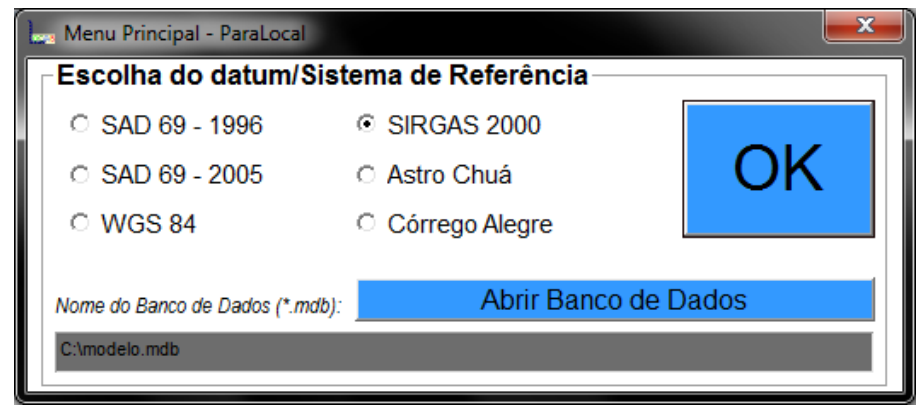

Figura 7: Menu de inserção de informações brutas e escolha do Banco de Dados. Fonte: Bruno Z. Prina (2014). 
A Figura 8 apresenta uma visualização geral do aplicativo ParaLocal (PRINA, 2014b). Assim, verificando o "Sistema Tabular", prosseguir-se-á ao próximo passo, no qual é a definição da coordenada base (latitude, longitude e altitude elipsoidal) para a conversão das coordenadas (para local). Dessa forma, a definição ocorre com um clique sobre uma das linhas dispostas no "Sistema Tabular".

$\mathrm{Na}$ parte mais abaixo do aplicativo, como pode ser visualizado na Figura 8, observa-se o "Registro da área calculada" (mensurado com as coordenadas locais convertidas). Deve-se ressaltar ainda, que com o botão "Adicionar ao histórico" é possível mover os dados para a "caixa de texto" que posteriormente pode ser salva como um documento na extensão “*.TXT", por meio do botão "Salvar em TXT".

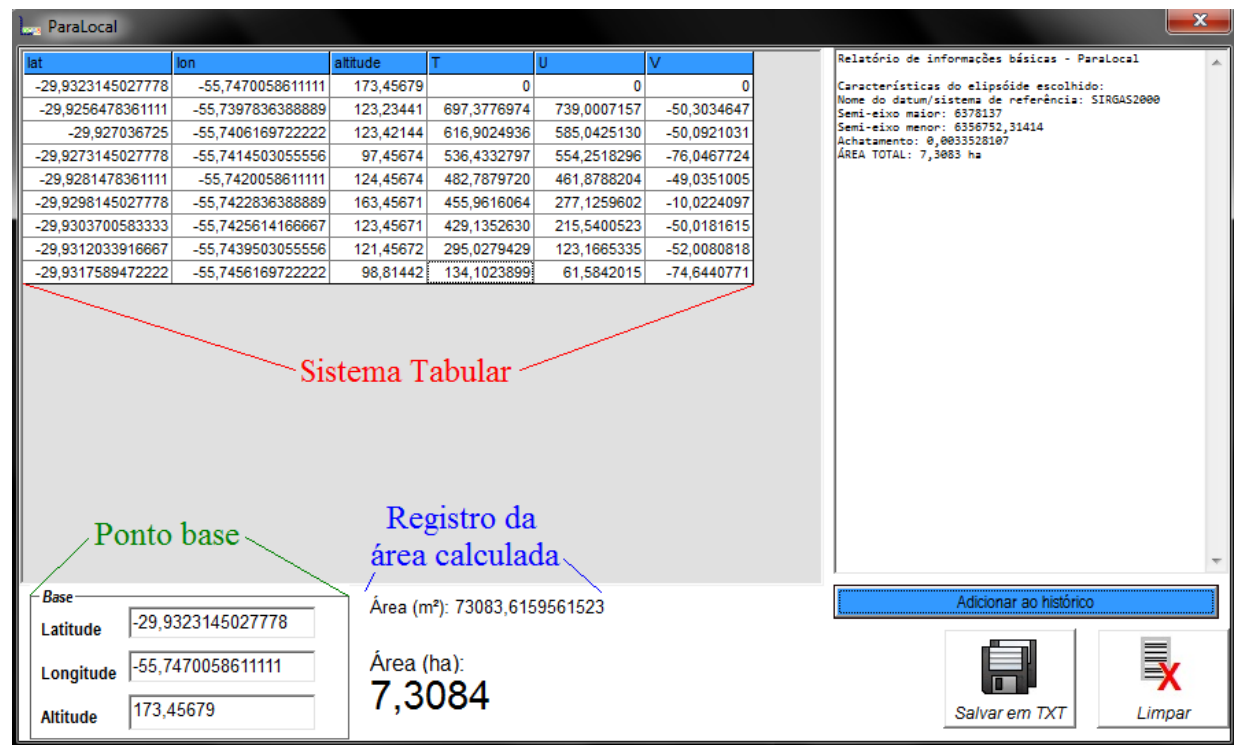

Figura 8: Análise geral do ParaLocal.

Fonte: Bruno Z. Prina (2014b).

Para a etapa de conversão das coordenadas, organizou-se a seguinte rotina:

Escolheram-se nove poligonais: três localizadas próximas a borda do fuso UTM lárea de ampliação - no município de Joia/RS), outras três próximas do centro do fuso (área de redução - no município de Uruguaiana) e as últimas três no local mais próximo possível da área em que o fator de escala é igual a 1 (no município de São Francisco de Assis) (conforme já visualizado na Figura 3);

Todas poligonais foram oriundas de levantamento de campo com apoio da tecnologia GNSS e foram pós-processadas no aplicativo Topcon Tools ${ }^{\circledR}$ (TOPCON TOOLS, 2014) e obtiveram 
precisões adequadas quanto à NTGIR em vigor (os dados utilizados foram obtidos através da empresa de topografia e georreferenciamento PROCAMPO, da cidade de Santa Maria/RS).

A partir dos dados pós-processados de cada levantamento, definiram-se as poligonais de análise, cada uma composta por 10 vértices, em três simulações geométricas: dispostas com alongamento norte-sul, alongamentos leste-oeste e regular (sem alongamento preferencial) (tentou-se definir as poligonais das formas mais adequadas possíveis, conforme a poligonal original).

Depois de organizado o processo de conversão das coordenadas e cálculo da área, realizouse a inserção dos dados das áreas, salvo anteriormente, para, que assim, fossem analisados os resultados, procedimento feito no Excel $^{\circledR}$ (MICROSOFT, 2007a).

Alerta-se que o padrão de referência dos dados, ou seja, aquela que serviu como a origem para a realização das porcentagens foi a área obtida pela metodologia do SIGEF (para obtenção da referida área, adaptou-se o aplicativo ParaLocal (PRINA, 2014b), a fim de calcular a área no padrão metodológico do SIGEF, ou seja, convertendo a poligonal original para local, utilizando como vértice-base o resultado da média aritmética de todos os vértices), que é a área calculada a partir das coordenadas locais, como ponto de referência (da conversão das coordenadas) o centro geométrico do polígono em questão. Assim, as áreas comparadas foram: a área obtida em coordenadas UTM, juntamente com as áreas máximas e mínimas quantificadas pelo aplicativo ParaLocal (PRINA, 2014b), com a simulação do cálculo de área com distintos ponto-base para a aplicação da matriz de rotação, conforme metodologia já explicitada.

Os valores "percentuais" possuíram uma taxa crescente de $100 \%$, a fim de destacar os números, mostrando-os mais significativos, com poucas casas decimais. Tal procedimento foi realizado pelo fato de que as discrepâncias obtidas foram pequenas, pois as variações existem, mas percentualmente em relação a área total mostram-se pouco significativas. Assim, a Equação 1 sistematiza o cálculo de ajustamento dos dados, os quais foram analisados.

$$
\text { Área } \% \text { ajustada }=\left(\frac{\text { Área } * 100}{\text { Área do SIGEF }}\right) * \text { fator de ajuste }
$$




\section{RESULTADOS E DISCUSSÕES}

A seguir é representada a estrutura geométrica das áreas nas quais foram aplicadas as etapas metodológicas do trabalho (Figura 9).

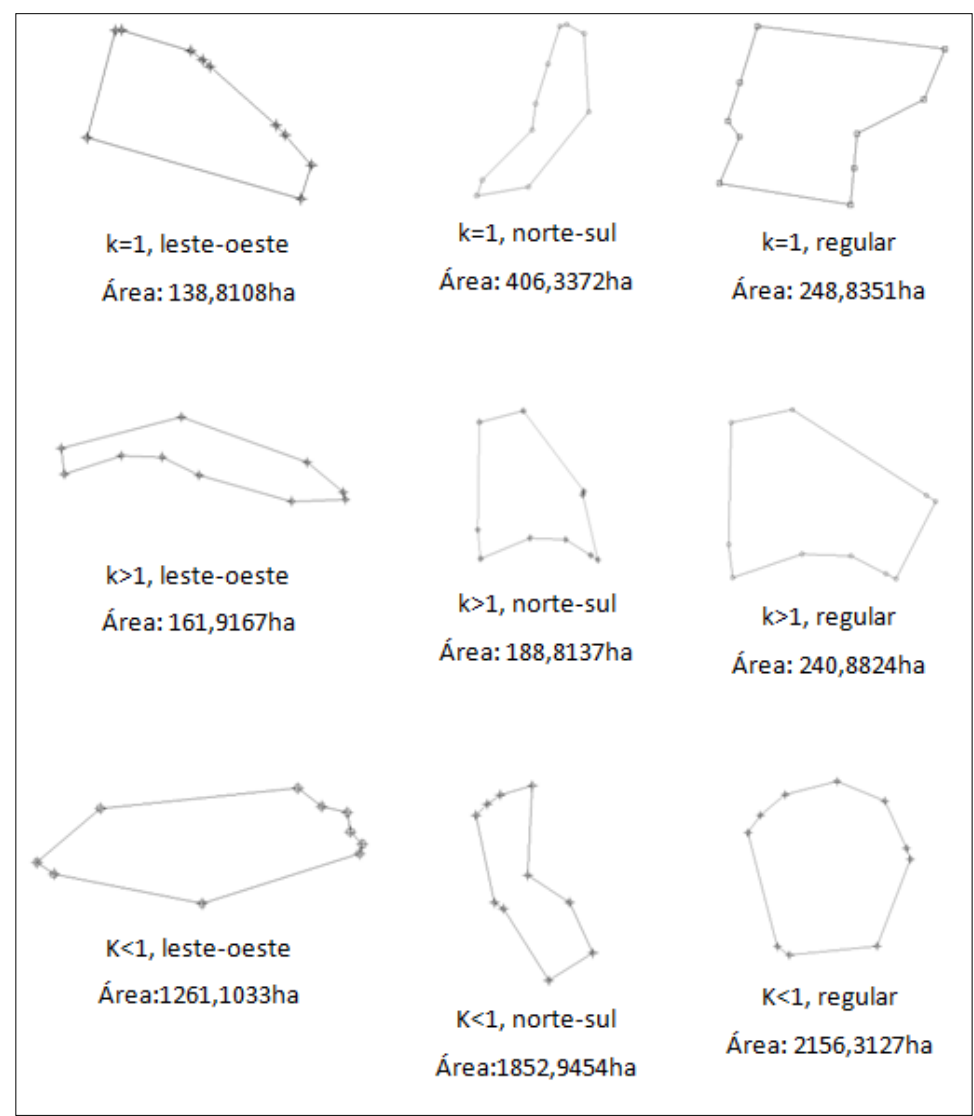

Figura 9: Poligonais analisadas com suas respectivas áreas calculadas sobre a projeção UTM. Fonte: Bruno Z. Prina (2014).

Destaca-se que juntamente com as análises principais, ocorreram mais três observações assim destacadas: a primeira referente à análise de uma poligonal o mais regular possível, a segunda com a poligonal exposta no sentido norte-sul, e por último uma poligonal no sentido leste-oeste. A partir da Figura 10, fica possível a identificação de vários fatores abordados neste trabalho. Assim, destaca-se, que para proceder da referida análise, inicialmente foram realizadas as comparações dos percentuais de áreas, e não os valores de superfície em hectares. Evidenciou-se que esta é a única forma de poder avaliar as discrepâncias, visto que as áreas das distintas poligonais geométricas possuíam valores diferentes (variando de 138 ha a 2156 ha, aproximadamente).

Ressalva-se, que na análise da Figura 10, ao aplicar o coeficiente de ajustamento da área (para extinguir a influencia do tamanho da área para com a variação, ou seja, utilizando a, 
porcentagem da mesma) fica visível que a área em UTM gera valores muito mais discrepantes para com as coordenadas locais.

Ainda, em relação à Figura 10, destaca-se, referenciando a área na projeção UTM, que a variação superficial nos locais com k=1 foram negativas, visto que a referida área não estava localizada bem no intervalo de secância do fuso UTM, fato que poderia ocorrer apenas com uma linha no sentido norte-sul do fuso, pois, após a linha de k=1 as deformações já ocorrem, aumentando gradativamente em relação ao centro do fuso (minimizando a área) e à sua borda (maximizando a área).

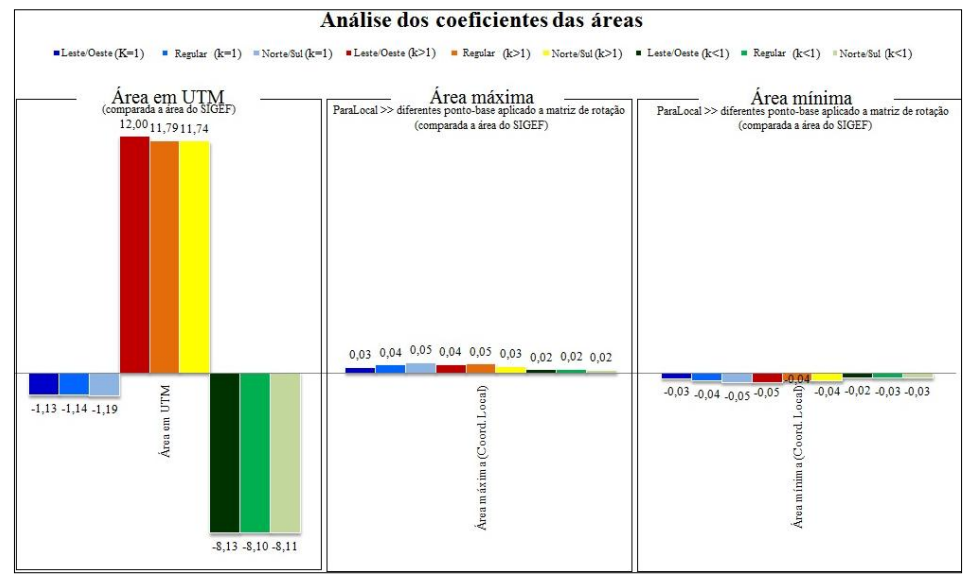

Figura 10: Resultado gráfico do processo investigativo: uso dos coeficientes das áreas: área percentual ajustada comparada à área do SIGEF.

Fonte: Bruno Z. Prina (2014).

Já na área de $k>1$, os valores, em média, foram de 0,1184\% maiores que a área obtida via metodologia do SIGEF, e no k<1, em média, houve uma redução de 0,0811\%. Intensifica-se, ainda, que não se mostrou significativo a forma da poligonal, pois os resultados não sofreram nenhuma correlação expressiva com esse âmbito.

Outra análise a ser enfatizada na Figura 10, refere-se à área oriunda das coordenadas locais. Destaca-se que ao avaliar as áreas máximas e mínimas (com o uso dos diferentes ponto-base para uso na matriz de rotação) registradas não se obtiveram discrepâncias significativas, pois, a disparidade mais acentuada foi de 0,0005 \%, para ambas direções (positiva e negativa). Destaca-se ainda que a forma da poligonal não foi significativa quanto aos parâmetros analisados neste estudo.

\section{Análises das coordenadas locais em diferentes regiões do fuso UTM}


Através da análise da variação das coordenadas locais, utilizando distintos pontos-base, ao longo do fuso UTM, constatou-se uma alta correlação entre a altitude do ponto-base para com a área gerada, sendo inversamente proporcional, ou seja, quanto maior a altitude, na maioria dos casos, menor a área mensurada, e assim vice e versa. Com o objetivo de justificar a afirmativa anterior, a seguir, apresentam-se as Figuras 11, 12 e 13, as quais demonstram a variação de área nos locais com k=1, as Figuras 14, 15 e 16 com k>1 e, por fim, as Figuras 17, 18 e 19 com k<1. Ressalta-se que para realização da correlação utilizou-se o coeficiente de correlação linear de Pearson $\left(R^{2}\right)$.

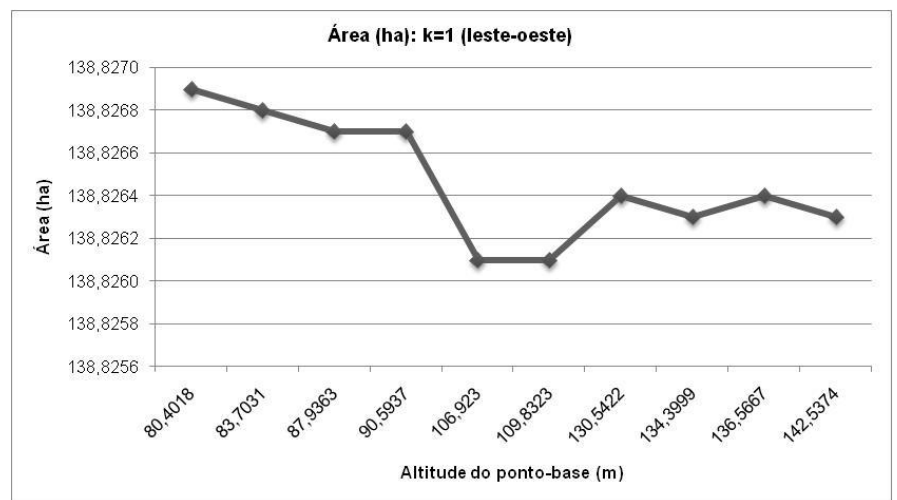

Figura 11: Valores superficiais quantificados para poligonal leste-oeste, com fator de escala próximo a k=1, que obteve $\mathrm{R}^{2}=-68,7 \%$.

Fonte: Bruno Z. Prina (2014).

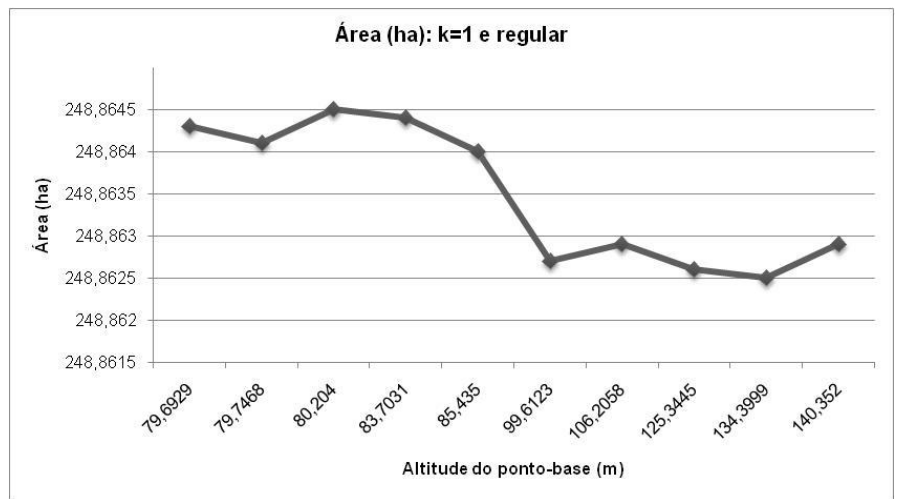

Figura 12: Valores superficiais quantificados para poligonal regular, com fator de escala próximo a k=1, que obteve $R^{2}=-86,3 \%$.

Fonte: Bruno Z. Prina (2014). 


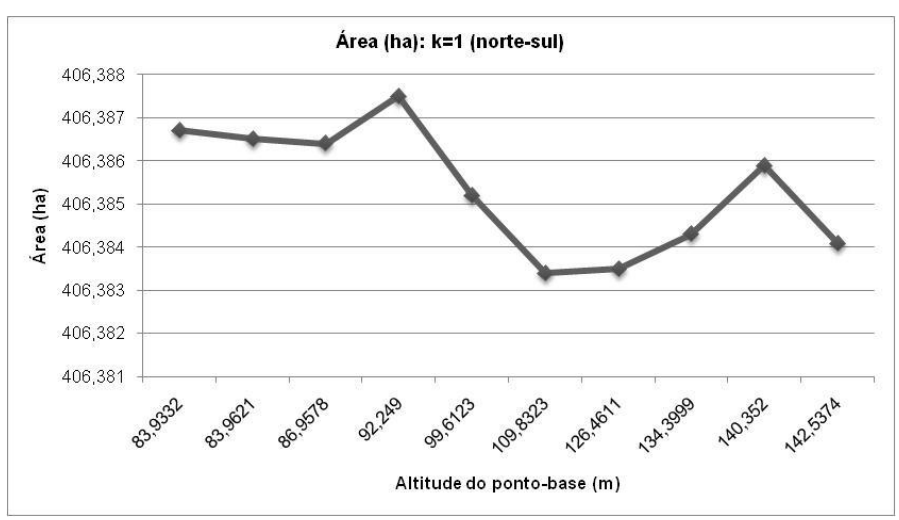

Figura 13: Valores superficiais quantificados para poligonal norte-sul, com fator de escala próximo a k=1, que obteve $R^{2}=-65,6 \%$

Fonte: Bruno Z. Prina (2014).

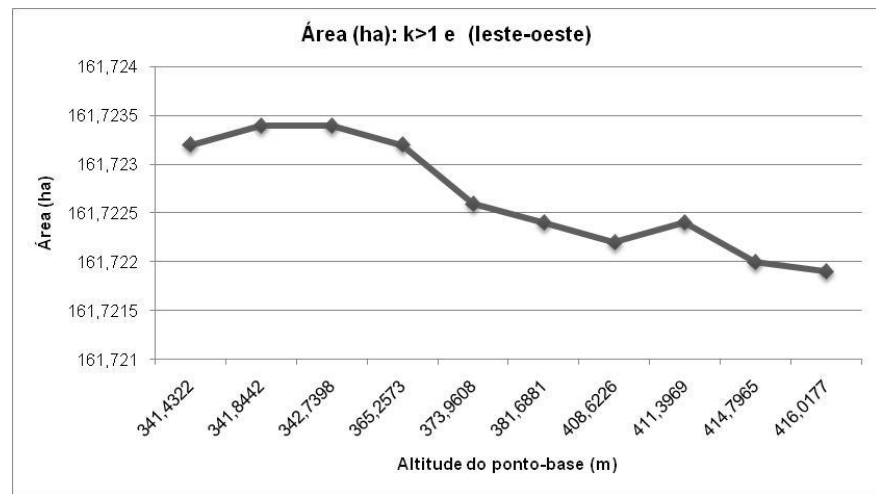

Figura 14: Valores superficiais quantificados para poligonal leste-oeste, com fator de escala próximo a k $>1$, que obteve $\mathrm{R}^{2}=-95 \%$.

Fonte: Bruno Z. Prina (2014).

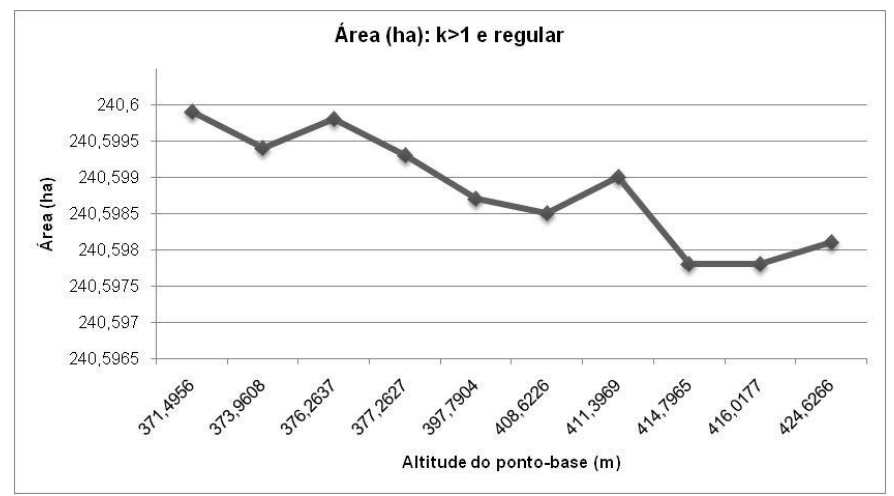

Figura 15: Valores superficiais quantificados para poligonal regular, com fator de escala próximo a k>1, que obteve $R^{2}=-87,6 \%$.

Fonte: Bruno Z. Prina (2014). 


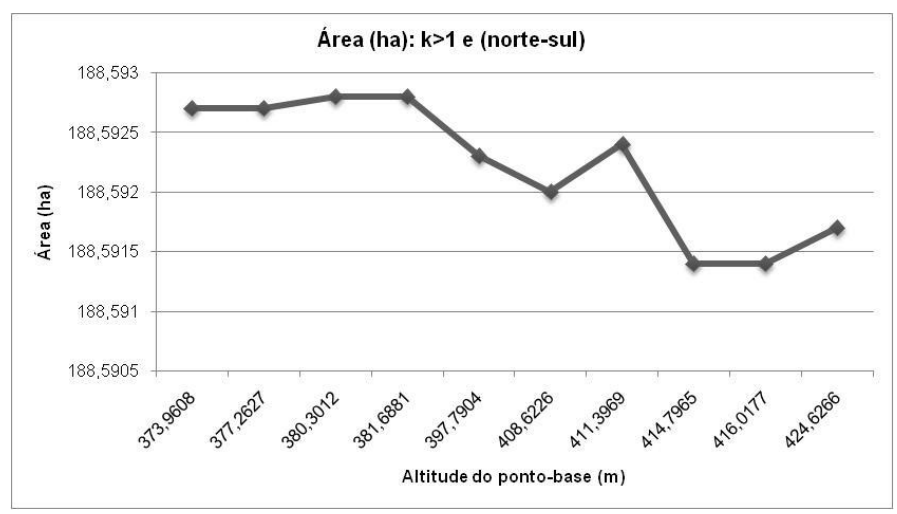

Figura 16: Valores superficiais quantificados para poligonal norte-sul, com fator de escala próximo a $k>1$, que obteve $R^{2}=-87,8 \%$.

Fonte: Bruno Z. Prina (2014).

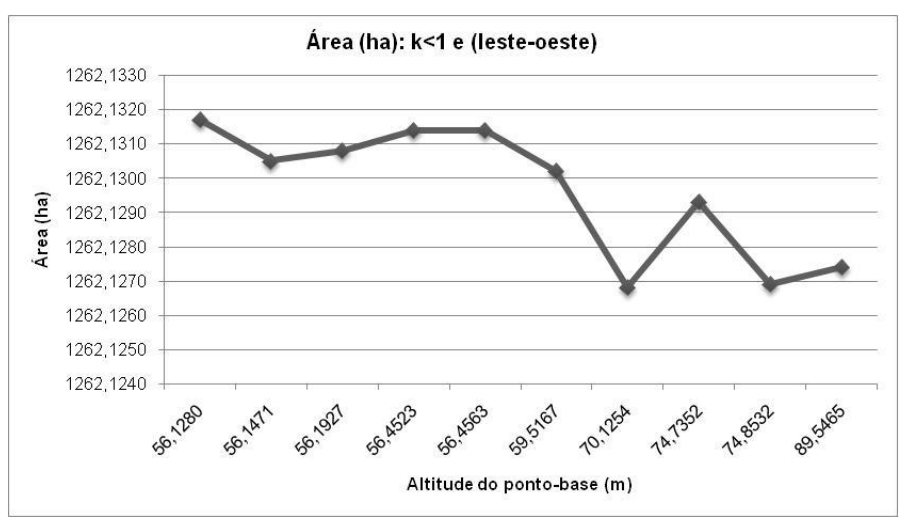

Figura 17: Valores superficiais quantificados para poligonal leste-oeste, com fator de escala próximo a $k<1$, que obteve $R^{2}=-83,2 \%$.

Fonte: Bruno Z. Prina (2014).

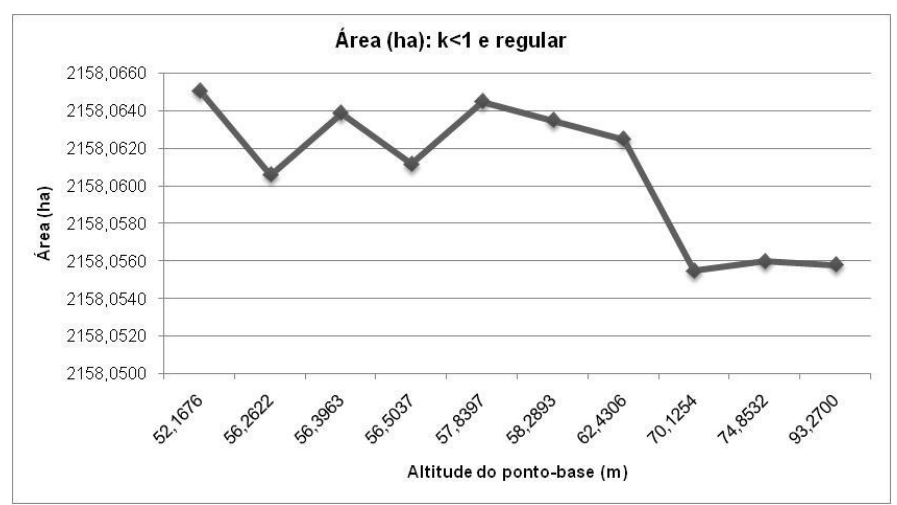

Figura 18: Valores superficiais quantificados para poligonal regular, com fator de escala próximo a $k<1$, que obteve $R^{2}=-82,1 \%$.

Fonte: Bruno Z. Prina (2014). 


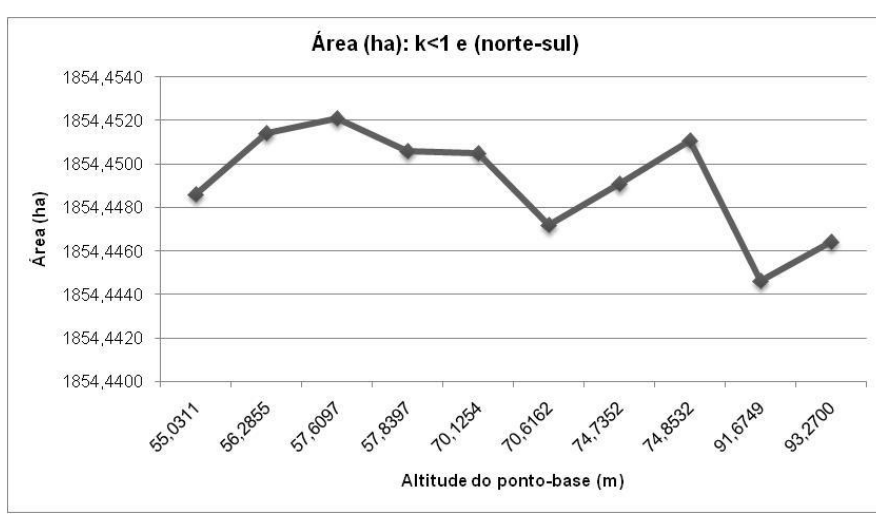

Figura 19: Valores superficiais quantificados para poligonal norte-sul, com fator de escala próximo a $k<1$, que obteve $R^{2}=-74,9 \%$.

Fonte: Bruno Z. Prina (2014).

Enfatiza-se que, analisando as correlações $\left(R^{2}\right)$, mostrou-se insignificante as interdependências entre a área gerada com: as formas das poligonais, bem como com a localização da área (no fuso UTM).

Após a análise dos dados descritos nas inúmeras figuras anteriores, constataram-se, por meio do Coeficiente de Correlação Linear de Pearson, as convergências existentes entre a variável altitude do ponto base e área da poligonal (em local) as quais estão descritas na Figura 20. Assim, são evidenciadas na íntegra, todas as correlações das áreas mensuradas, podendo avaliar a correlação obtida entre as áreas geradas com a altitude do ponto base, evidenciando o padrão obtido para essa correlação, a qual manteve-se uniforme, com valores variando entre $-65,6 \%$ a $-95,0 \%$.

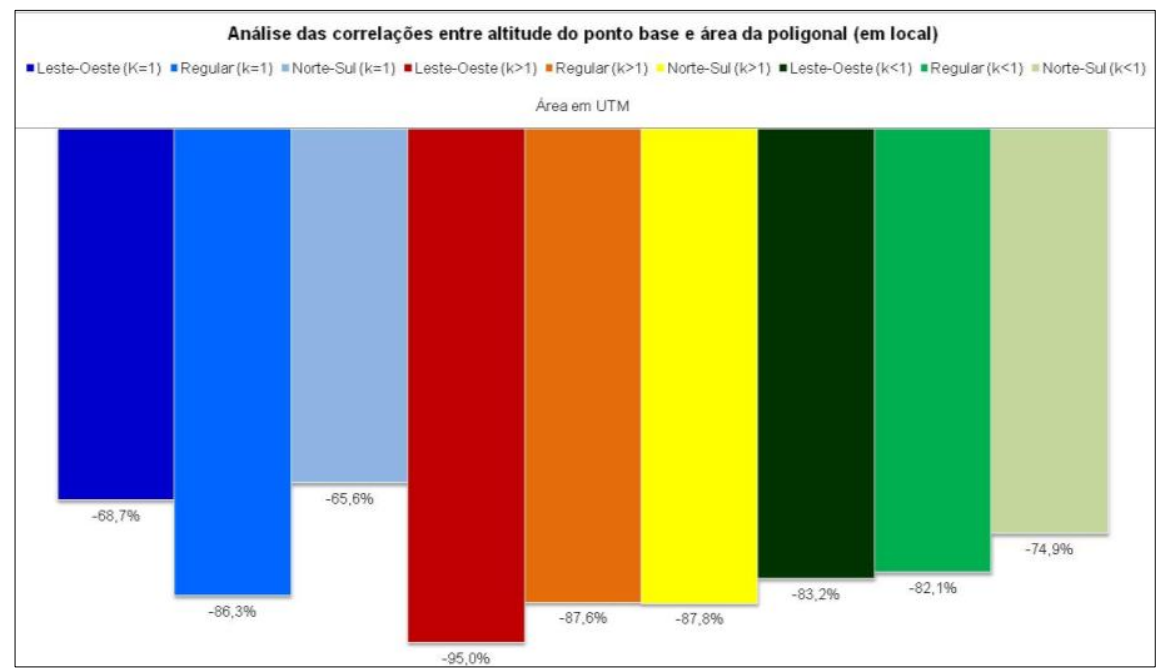

Figura 20: Análise das correlações entre altitude do ponto base e área da poligonal (em local) com o Coeficiente de Correlação Linear de Pearson.

Fonte: Bruno Z. Prina (2014). 


\section{Análise comparativa entre coordenadas locais e UTM em diferentes regiões do fuso UTM}

Com o foco de prover a comparação entre as coordenadas locais e UTM, verifica-se, por meio da Tabela 1, as variações da área calculada em coordenadas UTM, em coordenadas locais, bem como de suas discrepâncias.

\begin{tabular}{c|c|c|c|c|c}
\hline $\begin{array}{c}\text { Área em } \\
\text { UTM (ha) }\end{array}$ & $\begin{array}{c}\text { Área do } \\
\text { SIGEF } \\
\text { (ha) }\end{array}$ & $\begin{array}{c}\text { Discrepância } \\
\text { (ha) }\end{array}$ & $\begin{array}{c}\text { Fator de } \\
\text { ajuste: área da } \\
\text { UTM - área do } \\
\text { SIGEF }\end{array}$ & $\begin{array}{c}\text { Localização } \\
\text { no Fuso } \\
\text { UTM }\end{array}$ & $\begin{array}{c}\text { Forma da } \\
\text { poligonal }\end{array}$ \\
\hline 138,8108 & 138,8265 & $-0,0157$ & $-1,13$ & $\mathrm{k}=1$ & Leste-Oeste \\
\hline 248,8351 & 248,8635 & $-0,0284$ & $-1,14$ & $\mathrm{k}=1$ & Regular \\
\hline 406,3372 & 406,3854 & $-0,0482$ & $-1,19$ & $\mathrm{k}=1$ & Norte-Sul \\
\hline 161,9167 & 161,7227 & 0,194 & 12,00 & $\mathrm{k}>1$ & Leste-Oeste \\
\hline 240,8824 & 240,5988 & 0,2836 & 11,79 & $\mathrm{k}>1$ & Regular \\
\hline 188,8137 & 188,5922 & 0,2215 & 11,74 & $\mathrm{k}>1$ & Norte-Sul \\
\hline 1261,1033 & 1262,1298 & $-1,0265$ & $-8,13$ & $\mathrm{k}<1$ & Leste-Oeste \\
\hline 2156,3127 & 2158,0611 & $-1,7484$ & $-8,10$ & $\mathrm{k}<1$ & Regular \\
\hline 1852,9454 & 1854,4494 & $-1,504$ & $-8,11$ & $\mathrm{k}<1$ & Norte-Sul \\
\hline
\end{tabular}

Tabela 1: Análise geral das áreas em UTM e local (SIGEF).

Fonte: Bruno Z. Prina (2014).

Na Tabela 1, são verificadas, de forma quantitativa, as discrepâncias das coordenadas UTM para com a local (obtida pelo SIGEF), assim, nota-se que, de fato são mínimas as discrepâncias, porém, sobreleva-se, que as distorções da projeção UTM são visivelmente identificadas, com acréscimo de áreas nas regiões de k>1 e decréscimo de área com $k<1$. Com isso, pode-se afirmar que o calculo de área com coordenadas UTM apresenta valores incompatíveis para com o valor de fato que a área apresenta. Assim sendo, parabeniza-se os criadores da 3ㅇ NTGIR, pela mudança de metodologia de cálculo da área, pois, a partir dessa análise, os valores, de fato, mostrar-se-ão condizentes para com a realidade física superficial.

\section{CONSIDERAÇÕES FINAIS}

Considera-se muito relevante a metodologia de análise contida nesse trabalho, visto a grande exigência do conhecimento e compreensão das formas de cálculo de área, uma vez que o Georreferenciamento de Imóveis Rurais apresentou uma alteração na metodologia do cálculo de área. Assim sendo, o entendimento das diferenças envolvidas no cálculo de área com coordenadas UTM e locais são de profunda importância. Sobreleva-se que, por mais que foram obtidas pequenas variações nas áreas, é importante salientar que as precisões posicionais estabelecidas pelo INCRA são mínimas, logo, não adianta serem obtidas coordenadas precisas e acuradas, e realizar o cálculo da área em uma superfície fictícia, no caso das coordenadas UTM, as quais são secantes à superfície (em apenas dois vértices, 
quando $\mathrm{k}=1$ ), sendo, que a partir desses pontos as áreas tendem a serem, gradativamente, menores ou maiores.

A criação dos aplicativos FormaCoo (PRINA, 2014a) e ParaLocal (PRINA, 2014b) mostrou-se de suma relevância a esse estudo, visto que, todas as etapas metodológicas de análises das discrepâncias do cálculo de área foram compreendidas e explicadas.

A análise criteriosa das dissonâncias entre as áreas obtidas foi determinante para atingir, satisfatoriamente, todos os objetivos propostos. Assim sendo, conclui-se que o cálculo de área a partir das coordenadas UTM mostra-se incoerente para com a realidade topográfica local, pois, dependendo de sua localização espacial em um fuso UTM, as variações tendem a serem maiores ou menores, notificando que a área calculada em UTM não é a mesma que de fato existe no campo.

A análise do cálculo de área, em coordenadas locais, com distintos pontos-base, resultou em um estudo pioneiro de muita importância, pois foi desvendado que quanto maior a altitude do referido ponto, menor é a área gerada, na maioria das vezes. Tal afirmação foi possível, após verificar, exaustivamente, os Coeficientes de Correlação Linear de Pearson.

Ainda, cita-se que a análise das áreas com diferenças das formas geométricas mostrou-se pouco significativas, com variações sem um padrão de correlação.

Em relação à metodologia de cálculo de área realizada pelo SIGEF (2014), os autores mostram-se inteiramente de acordo com a mesma, visto duas questões. A primeira, pelo fato de ter como origem do cálculo as coordenadas locais. A segunda refere-se a importância de que o vértice utilizado para realizar a conversão para coordenadas locais é um ponto com valores médio dos pontos (nos três eixos cartesianos). Assim, enfatiza-se que, principalmente, a altitude terá um valor intermediário, ocasionando menor discrepância, conforme os dados descritos nesse trabalho.

Destaca-se, ainda, que a metodologia englobada nesse trabalho ajuda a compreender a dinâmica do calculo de área, bem como auxilia na forma de desenvolver as condutas dos trabalhos a campo. 
Por fim, em trabalhos posteriores, será aplicada essa mesma metodologia para figuras geométricas com maiores discrepâncias (regulares e irregulares) e com maior contraste entre os valores de áreas.

\section{AGRADECIMENTOS}

Agradecemos a CAPES (Coordenação de Aperfeiçoamento de Pessoal de Nível Superior) pela concessão da bolsa de estudos ao primeiro autor.

\section{BIBLIOGRAFIA}

BATALHA, G. de S. O uso de compilador em ambiente de aprendizagem de algoritmos. Dissertação apresentada ao Programa de Pós-graduação em Educação da Universidade Estácio de Sá como requisito parcial para obtenção do título de Mestre em Educação, Rio de Janeiro, 2008.

BRASIL. Portaria $n^{\circ} 486.2$ de setembro de 2013. Disponível em: <https://sigef.incra.gov.br/static/documentos/portaria_486.pdf>. Acesso em 29 mar.14.

DAL'FORNO, G. L., AGUIRRE, A. J.; HILLEBRAND, F. L.; GREGÓRIO,b F. de V. Transformação de coordenadas geodésicas em coordenadas no plano topográfico local pelos métodos da norma NBR 14166:1998 e o de rotações e translações. III Simpósio Brasileiro de Ciências Geodésicas e Tecnologias da Geoinformação. Recife - PE, 27-30 de Julho de 2010. Disponível em:

<http://www.ufpe.br/cgtg/SIMGEOIII/IIISIMGEO_CD/artigos/Cad_Geod_Agrim/Geodesia\%2 0e\%20Agrimensura/A_62.pdf>. Acesso em 23 mar.14.

DAL'FORNO, G. L.; AGUIRRE, A. J.; HILLEBRAND, F. L. GREGÓRIO, F. de V. Transformação de coordenadas geodésicas em coordenadas no plano topográfico local pelos métodos da norma NBR 14166/1998 e o de rotações / translações. Revista A Mira - Agrimensura e Cartografia, 2011. Disponível em: <http://www.amiranet.com.br/artigo/transformacao-decoordenadas-geodesicas-em-coordenadas-no-plano-topografico-local-pelos-metodos-danorma-nbr-14166-1998-e-o-de-rotacoes-translacoes-74>. Acesso em 27 mar.14.

GALVÃO, L. C. Apostila de Matemática Aplicada. Universidade Tecnológica Federal do Paraná UTFPR. Disponível em: <http://ce.esalq.usp.br/arquivos/aulas/2013/LCE0176/mat_aplicada_a.pdf>.

Acesso em 29 set.2014.

INCRA - Instituto Nacional de Colonização e Reforma Agrária. Norma Técnica para o Georreferenciamento de Imóveis Rurais. Aplicada à Lei 10.267, de 28 de agosto de 2001 e do Decreto 4.449, de 30 de outubro de 2002. 1ạ Edição, novembro/2003.

INCRA - Instituto Nacional de Colonização e Reforma Agrária. Norma Técnica para o Georreferenciamento de Imóveis Rurais. Aplicada à Lei 10.267, de 28 de agosto de 2001 e do Decreto 4.449, de 30 de outubro de 2002. 2a Edição, fevereiro/2010a. 
INCRA - Instituto Nacional de Colonização e Reforma Agrária. Norma Técnica para o Georreferenciamento de Imóveis Rurais. Aplicada à Lei 10.267, de 28 de agosto de 2001 e do Decreto 4.449, de 30 de outubro de 2002. 2a Edição Revisada, agosto/2010b.

INCRA - Instituto Nacional de Colonização e Reforma Agrária. Norma Técnica para o Georreferenciamento de Imóveis Rurais. 3a Edição, Brasília, 2013a. Disponível em: $<$ https://sigef.incra.gov.br/static/documentos/norma_tecnica_georreferenciamento_imovei s_rurais_3ed.pdf>. Acesso em 29 mar.14.

INCRA - Instituto Nacional de Colonização e Reforma Agrária. Manual Técnico de Posicionamento. 1a adição, Brasília, 2013b. Disponível em: <https://sigef.incra.gov.br/static/documentos/manual_tecnico_posicionamento_1ed.pdf $>$. Acesso em 29 mar.14.

INCRA - Instituto Nacional de Colonização e Reforma Agrária. Manual Técnico de Limite e Confrontações. 1a Edição, Brasília, 2013c. Disponível em: <https://sigef.incra.gov.br/static/documentos/manual_tecnico_limites_confrontacoes_1ed. pd>. Acesso em 29 mar.14.

INCRA - Instituto Nacional de Colonização e Reforma Agrária. Manual para Gestão da Certificação de Imóveis Rurais. 1ạ Edição, Brasília, 2013d. Disponível em: <https://sigef.incra.gov.br/static/documentos/manual_gestao_certificacao_1ed.pdf>.

Acesso em 29 mar.14.

MICROSOFT. Microsoft ${ }^{\circledR}$ Office Word, 2007a.

MICROSOFT. Microsoft ${ }^{\circledR}$ Office Access, 2007b.

PRINA B. Z. FormaCoo. Aplicativo desenvolvido com a linguagem de programação Visual Basic. 2014a.

PRINA B. Z. ParaLocal. Aplicativo desenvolvido com a linguagem de programação Visual Basic. 2014b.

SAMPAIO, E. Noções de Cartografia. Departamento de Geociências, Universidade de Évora, 2005. Disponível em: <http://home.dgeo.uevora.pt/ ems/files/Anexo\%20B-05.PDF>. Acesso em 23 mar.14.

SIGEF. Sistema de Gestão Fundiária. 2014. Disponível em: <https://sigef.incra.gov.br/>. Acesso em 29 mar.14.

SILVA, A. M. da; NERY, A. M. de C.; GONCALVES, M. L. A. M.; NERO, M. A. . Análise comparativa quantitativa de metodologias de conversão de coordenadas topográficas arbitrária para coordenadas UTM. In: VIII Colóquio Brasileiro de Ciências Geodésicas, 2013, Curitiba. Anais do VIII Colóquio Brasileiro de Ciências Geodésicas. Curitiba-PR: Universidade Federal do Paraná, 2013. v. 1. p. 1-1.

TOPCON TOOLS. Versão 7.5.1, Copyright 2003-2009, Topcon Positioning Systems, Inc, 2014.

\begin{tabular}{cc} 
Artigo submetido em & $21 / 07 / 2014$ \\
\hline Artigo aceito em & $20 / 03 / 2015$
\end{tabular}

